\section{Abstract E-025 Table 1}

Tandem lesion

$(\mathrm{N}=55)$

Baseline characteristics

Age, years (mean (SD))

Female

$67.8(10.4)$

Medical history

Atherosclerosis

Atrial fibrillation

Diabetes

Hypertension

Time from onset to hospital admission, hrs (median [IQR])

ASPECTS (median [IQR])

NIHSS (median [IQR])

IV tPA pre-procedure

Procedural metrics

Degree of stenosis in proximal (tandem) lesion,\% (median [IQR])

First line of treatment in proximal (tandem) lesion

Angioplasty only

Aspiration only

$36.4 \%$

Stent

Additional treatment in proximal (tandem) lesion

Balloon angioplasty

Stent

First line of treatment in intracranial vessel

Direct aspiration alone

Direct aspiration with 3D Revascularization Device

$14.5 \%$

$18.2 \%$

$29.1 \%$

$67.3 \%$

$2.7[1.6-5.9]$

7 [6-9]

15 [9-20]

$61.8 \%$

99 [78-100]

Number of total passes in all locations

3

$4+$

Time from arterial puncture to $\mathrm{mTICl} 2 \mathrm{~b}-3$ else final angiogram, min

(median [IQR])

Primary endpoints

$\mathrm{mTICl} 2 \mathrm{~b}-3$ post-procedure

mRS $0-2$ at 90 days

All-cause mortality at 90 days

Secondary endpoints

Device-related SAEs, $\leq 24$ hrs

Device-related SAEs, overall

Procedure-related SAEs, $\leq 24$ hrs

Procedure-related SAEs, overall

ENT at end of procedure

Symptomatic ICH at 24 hrs

Vessel perforation

Vessel dissection

$89.1 \%$

$63.5 \%$

$7.3 \%$

$1.8 \%$

$3.6 \%$

$9.1 \%$

$14.5 \%$

$5.5 \%$

$7.3 \%$

$1.8 \%$

$5.5 \%$

\title{
E-026 UTILIZATION OF A LARGE-BORE 088 INTRACRANIAL ACCESS CATHETER FOR COMPLEX NEUROINTERVENTION: EXPERIENCE WITH THE TRACSTAR LDP ${ }^{\text {TM }}$ IN 127 CONSECUTIVE CASES
}

${ }^{1} \mathrm{~J}$ Campos*, ${ }^{1} \mathrm{Z}$ Hsu, ${ }^{2} \mathrm{D}$ Zarrin, ${ }^{1} \mathrm{~K}$ Golshani, ${ }^{3} \mathrm{~N}$ Beaty, ${ }^{4} \mathrm{M}$ Bender, ${ }^{2} \mathrm{G}$ Colby, ${ }^{5} \mathrm{~L}$ Lin, ${ }^{5} \mathrm{~A}$ Coon. 'Department of Neurological Surgery, University of California, Irvine, Orange, CA; ${ }^{2}$ Department of Neurosurgery, University of California, Los Angeles, Los Angeles, CA; ${ }^{3}$ Department of Neurosurgery, Tallahassee Neurological Clinic, Tallahassee, FL; ${ }^{4}$ Department of Neurosurgery, University of Rochester Medical Center, Rochester, NY; ${ }^{5}$ Department of Neurosurgery, Carondelet Neurological Institute, St. Joseph's Hospital, Tucson, AZ

Background The delivery of complex neuroendovascular devices requires a robust proximal access platform. This demand has previously been met with a $6 \mathrm{Fr}$ long-sheath ( $8 \mathrm{Fr}$ guide) that is placed in the proximal ICA (high cervical) or vertebral artery (V1/2) segments. We share our experience with the first 088 8Fr guide catheter designed specifically for direct intracranial access.

Methods We retrospectively reviewed a prospectively-maintained IRB-approved institutional database of the senior authors to identify all cases where the TracStar LDP (Imperative Care, Campbell, CA) was utilized. Case details were recorded including patient demographics, case type, and distal location achieved with navigation of the guide catheter. Technical success was defined as safe placement of the guide catheter to at least the petrous (anterior circulation) or vertebral artery (V3) segments (posterior circulation) or the transverse sinus for venous stenting procedures.

Results Over the 14-month study period, 127 femoral access cases were identified where the TracStar LDP guide catheter was utilized for aneurysm treatment $(80 \%$ of cases, $102 / 127$ ), intracranial angioplasty/stenting (13\% of cases, 17/127), venous sinus stenting (3\% of cases, 4/127), and other pathologies (3\% of cases, 4/127). All cases utilized a direct select catheter technique for placement (no exchange). Posterior circulation placement occurred in $14 \%$ of cases $(18 / 127)$. Technical success was achieved in $97 \%$ of cases $(123 / 127)$. Of the 4 cases with technical failure, $75 \%(3 / 4)$ were in the posterior circulation and were attributed to tortuous proximal subclavian access or a small caliber vertebral origin. No symptomatic or flow-restricting vessel dissections occurred in any of the cases.

Conclusion The TracStar LDP is a safe and effective means for establishing direct 088 intracranial access. This can be achieved in a wide range of cases with extremely high rates of technical success.

Disclosures J. Campos: None. Z. Hsu: None. D. Zarrin: None. K. Golshani: None. N. Beaty: 2; C; Medtronic Neurovascular, Stryker Neurovascular. M. Bender: None. G. Colby: 2; C; Medtronic Neurovascular, MicroVention-Terumo, Stryker Neurovascular. L. Lin: 2; C; Medtronic Neurovascular, MicroVention-Terumo, Stryker Neurovascular. A. Coon: 2; C; Avail MedSystems, Imperative Care, InNeuroCo, Medtronic Neurovascular, MicroVention-Terumo, Q'apel, Rapid Medical, Stryker Neurovascular.

\section{E-027 TRANS-FEMORAL 5FR DIAGNOSTIC CATHETER ANGIOGRAPHY FROM A 4FR ACCESS USING A 5/4 SLENDER RADIAL SHEATH: EXPERIENCE IN 50 CONSECUTIVE CASES}

${ }^{1} \mathrm{~J}$ Campos*, ${ }^{1} \mathrm{Z}$ Hsu, ${ }^{2} \mathrm{D}$ Zarrin, ${ }^{1} \mathrm{~K}$ Golshani, ${ }^{3} \mathrm{~N}$ Beaty, ${ }^{4} \mathrm{M}$ Bender, ${ }^{2} \mathrm{G}$ Colby, ${ }^{5} \mathrm{~L}$ Lin, ${ }^{5} \mathrm{~A}$ Coon. ${ }^{1}$ Department of Neurological Surgery, University of California, Invine, Orange, $C A$; ${ }^{2}$ Department of Neurosurgery, University of California, Los Angeles, Los Angeles, CA; ${ }^{3}$ Department of Neurosurgery, Tallahassee Neurological Clinic, Tallahassee, FL; ${ }^{4}$ Department of Neurosurgery, University of Rochester Medical Center, Rochester, NY; ${ }^{5}$ Department of Neurosurgery, Carondelet Neurological Institute, St. Joseph's Hospital, Tucson, AZ

\subsection{6/neurintsurg-2021-SNIS. 123}

Background Femoral access has long been the standard for diagnostic angiography but recently radial access techniques have gained popularity due to their perceived enhanced safety. The slender $5 / 4$ radial sheath (5/4RS) (Terumo, Japan) is the commonly used sheath for radial access that allows the use of 